\title{
A THEOREM ON GENERIC NORMS OF STRICTLY POWER ASSOCIATIVE ALGEBRAS
}

J. TITS

Let $A$ be a finite-dimensional strictly power associative algebra with an identity element over an arbitrary field $k$, and let

$$
m(x)=x^{n}+\sum_{i=1}^{n} \lambda_{i}(x) x^{n-i}
$$

be its generic minimum polynomial [1]. The coefficients $\lambda_{i}$ are polynomial functions on $A$. Such a function $f$ is called a Lie invariant under a linear transformation $L$ of the underlying vector space if $f(a+t L(a)) \equiv f(a)\left(\bmod t^{2}\right)$ where $t$ is an indeterminate and $f$ is extended in the usual way to the vector space over $k(t)$; in particular, if $f$ is a linear form on $A$ (for instance the generic trace $\lambda_{1}$ ), this means that $f(L(a))=0$.

THEOREM. The coefficients $\lambda_{i}$ of the generic minimum polynomial are Lie invariant under every derivation $d$ of $A$.

Assuming that $A$ is a Jordan algebra (over a field of characteristic not two), that $i=1$ and that $d$ is the inner derivation which sends $a$ into $b \cdot a c-b a \cdot c$, we have the

Corollary. The identity $\lambda_{1}(b \cdot a c)=\lambda_{1}(b a \cdot c)$ holds in any Jordan algebra.

This result has been obtained independently by N. Jacobson (unpublished).

Proof of the theorem. Let $K$ be an arbitrary extension of $k$. The extensions of the forms $\lambda_{i}$ and of the derivation $d$ to $A_{K}$ will be denoted by the same symbols $\lambda_{i}$ and $d$. Let $t$ be an indeterminate scalar and, for $a, b \in A_{K}$, denote by $\{a, b\}_{i}$ (resp. $\left.\mu_{i}(a, b)\right)$ the coefficient of $t$ in $(a+t b)^{i}$ (resp. in $\left.\lambda_{i}(a+t b)\right)$. As $m(a+t b)$ vanishes identically, the coefficient of $t$ in it must be zero, that is,

$$
\{a, b\}_{n}+\sum_{i=1}^{n} \lambda_{i}(a) \cdot\{a, b\}_{n-i}+\sum_{i=1}^{n} \mu_{i}(a, b) \cdot a^{n-i}=0
$$

It is easily seen that $d\left(a^{i}\right)=\{a, d(a)\}_{i}$; therefore

Received by the editors October 1, 1962. 
(2)

$$
d(m(a))=\{a, d(a)\}_{n}+\sum_{i=1}^{n} \lambda_{i}(a) \cdot\{a, d(a)\}_{n-i}=0
$$

for every $a \in A_{K}$. Setting $b=d(a)$ in (1) and subtracting (2), we have

$$
\sum_{i=1}^{n} \mu_{i}(a, d(a)) \cdot a^{n-i}=0 .
$$

If $a$ is generic (over $k$ ), it does not satisfy any polynomial identity of order $n-1$, with coefficients in $K$; thus

$$
\mu_{i}(a, d(a))=0,
$$

and the same relations then hold for arbitrary $a \in A_{K}$.

By the definition of the $\mu_{i}(a, b)$ this is the Lie invariance of the $\lambda_{i}(a)$ which we wished to prove.

\section{BibliogRAPHY}

1. N. Jacobson, Some groups of transformations defined by Jordan algebras. I, J. Reine Angew. Math. 201 (1959), 178-195.

Universite de BruXelles, BruXelles, Belgium 